\title{
NEAR INFRARED STAR COUNTS AS A PROBE OF ASYMMETRIES IN THE GALAXY'S DISK
}

\author{
JAMES E. RHOADS \\ Princeton University Observatory, Princeton NJ 08544, USA. \\ email: rhoads@astro.princeton.edu
}

I have recently started an observing program to study the distribution of dynamically old giant stars in the disk, looking for (i) large amplitude spiral arms and (ii) small amplitude corrugations.

(i) Theoretical studies of spiral density waves usually assume that the mass density contrast is weak and the waves linear. Recent observations in red and near-IR light (e.g., Elmegreen and Elmegreen 1984; Rix and Zaritsky 1994), coupled with stellar population models, suggest otherwise. In the Galaxy, we can compare observations of diffuse NIR light and of individual stars, eliminating the need for population modeling. Moreover, we can estimate distances to stars to learn about 3D structure.

(ii) Recent work by Malhotra (1994a; 1994b) has shown that the midplane of the molecular and atomic gas differs from the nominal (Galactic latitude $b=0^{\circ}$ ) plane by up to $50 \mathrm{pc}$ in the inner Galaxy. Various observations (Djorgovski \& Sosin 1989, Freudenreich et al 1994, Carney \& Seitzer 1993) show that the stellar distribution also deviates from $b=0$. We will choose fields to test the possibility that the stars behave like the gas. This constrains theories of the corrugations; e.g., if old stars participate then magnetic fields aren't the cause.

We use the $3.5 \mathrm{~m}$ telescope at Apache Point Observatory in NIR and optical bands. We use giant stars as tracers; they are common, can be seen to distances $\sim 17 \mathrm{kpc}$, and have a fairly narrow luminosity function (FWHM $\sim 2 \mathrm{mag}$, cf. table 2 of Wainscoat et al 1992) so are somewhat useable as standard candles. At fixed apparent magnitude, giant stars are further away than cool dwarfs. They will therefore suffer more reddening and will lie in a different region of the CMD (cf. figure 1).

Further details on the methods can be found in Rhoads 1995. 

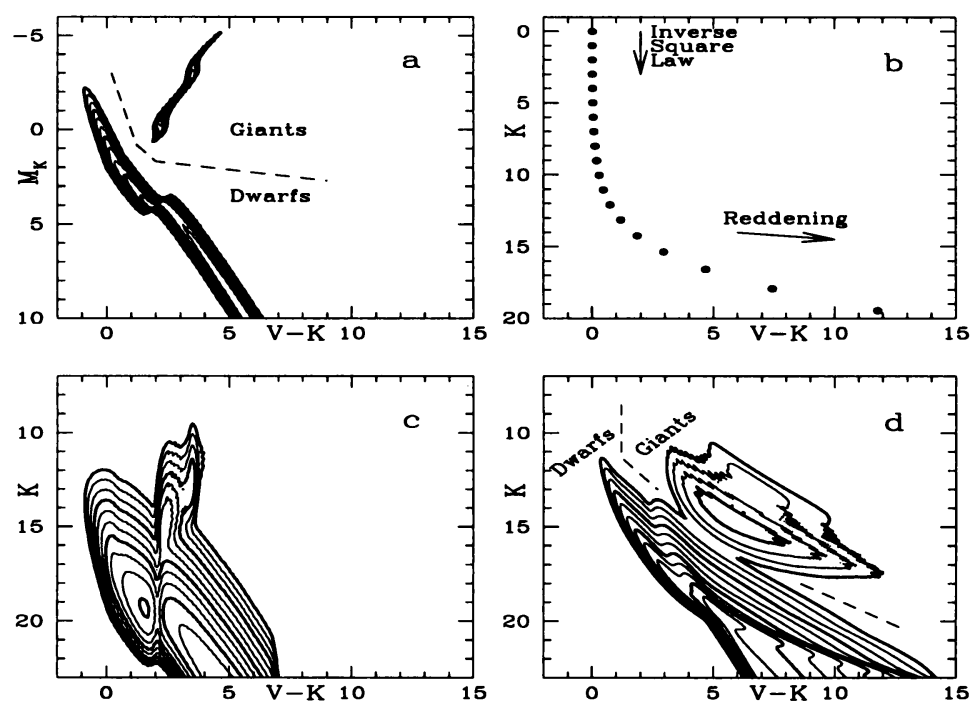

Figure 1. Model stellar densities in $K,(V-K)$ color-magnitude diagrams illustrate ou] sample selection procedure. Contours are logarithmically spaced by factors of $10^{1 / 3}$; ev. ery third contour is emphasized. (a) Contours are for unreddened stars at $d=10 \mathrm{pc}$. (b) The reddening vector for $A(V)=0.5 \mathrm{mag}$ is shown. The solid circles mark the location o: an $\mathrm{A} 0 \mathrm{~V}$ star at distance moduli $0,1,2, \ldots$, assuming extinction $A_{V}=0.33 \mathrm{mag} / \mathrm{kpc}$ (c) CMD for $l=60^{\circ}, b=0^{\circ}$ assuming no extinction. The lowest contour level is $1 \mathrm{star} /\left[\left(2^{\prime}\right)^{2}(K \mathrm{mag})(V-K \mathrm{mag})\right]$. (d) As (c), but assuming $A_{V}=0.33 \mathrm{mag} / \mathrm{kpc}$.

\section{Acknowledgements}

I thank my advisor David Spergel and the Princeton area observing com. munity for discussion and advice. Also, I thank Richard Wainscoat for his model of the IR sky. Financial support for this work is provided by NSF grant AST91-17388 and by NASA grant NAG5-2693.

\section{References}

Carney, B. W. \& Seitzer, P. 1993. AJ 105, 2127.

Djorgovski \& Sosin 1989. ApJ Lett 341, L13.

Elmegreen, D. M. \& Elmegreen, B. G. 1984. ApJS 54, 127.

Freudenreich et al 1994. ApJ Lett 429, L69.

Malhotra, S. 1994a. ApJ 433, 687.

Malhotra, S. 1994b. ApJ, submitted.

Rhoads, J. E. 1995. In the proceedings of the "Formation of the Milky Way" workshop E. Alfaro, ed., Cambridge University Press.

Rix, H.-W. \& Zaritsky, D. 1994. ApJ, submitted.

Wainscoat, R. J. et al 1992. ApJS 83, 111. 